\title{
Erratum to: Coral Reefs of the World
}

\section{Hajime Kayanne}

(C) Springer Japan 2016

H. Kayanne (ed.), Coral Reef Science, Coral Reefs of the World 5, DOI 10.1007/978-4-431-54364-0

DOI 10.1007/978-4-431-54364-0

DOI 10.1007/978-4-431-54364-0_1

DOI 10.1007/978-4-431-54364-0_2

DOI 10.1007/978-4-431-54364-0_7

DOI 10.1007/978-4-431-54364-0_8

The following corrections were made after original publication

Page 26, lines 9 and 10 (right column): bad line break

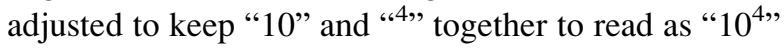

Page iv, cover caption: Bleached coral colony (Porites cyrindrica) changed to Bleached coral colony (Porites cylindrica)

Page $32,4^{\text {th }}$ and $5^{\text {th }}$ lines from the bottom (left column): bad line break adjusted to keep "ml" and "-1" together to read as " $\mathrm{ml}^{-1}$ ",

Page 6, line 4 (left column) and page 24 line 6 (right column): Wewenkang changed to Wewengkang

Page 35, line 2 (left column): bad line break adjusted to keep "h" and "-1" together to read as " $\mathrm{h}$ "1"

Page 22, line 19 (right column): Platax 12: 1-9 changed to Platax 12: 19-27

Page $84,3^{\text {rd }}$ line from the bottom (left column), '55-year survey' was changed to '15-year survey'

The updated original online version for this book can be found at

DOI 10.1007/978-4-431-54364-0

DOI 10.1007/978-4-431-54364-0_1

DOI 10.1007/978-4-431-54364-0_2

DOI 10.1007/978-4-431-54364-0_7

DOI 10.1007/978-4-431-54364-0_8

H. Kayanne

Department of Earth and Planetary Science, Graduate School of Science,

The University of Tokyo, Tokyo, Japan

e-mail: kayanne@eps.s.u-tokyo.ac.jp 
Page 84, Fig. 7.3 caption revised to read as 'Locality map for examining relation between bleaching events and SST anomalies. Isolines show number of coral genera'

Page 92, Fig. 7.9 caption revised to read as 'Feedback loops between global stresses and coral and coral reefs. Solid lines: positive couplings; dashed lines: negative couplings. P and $\mathrm{N}$ denote positive and negative feedbacks, respectively'

Page 96, lines 33 and 34 (right column), bad line break adjusted to keep " $\delta$ " and " $H$ " together to read as
" $\delta^{2} \mathrm{H}$ ", also space inserted around minus sign to read as " $\delta^{2} \mathrm{H}-8 \delta^{18} \mathrm{O}$

Page 96, lines 49-50 (right column), bad line break adjusted to keep " $\delta$ " and " $\mathrm{H}$ " together to read as " $\delta{ }^{2} \mathrm{H}$ "

Page 97, lines 7 and 8 (left column), bad line break adjusted to keep comma and $\mathrm{NO}_{3}{ }^{-}$together

Page 98, lines 18 and 19 (right column): bad line break adjusted to keep " $\mathrm{NO}_{3}$ " and "-" together to read as " $\mathrm{NO}_{3}{ }^{-}$" Page 98, lines 22 and 23 (right column): bad line break adjusted to keep " $\mathrm{L}$ " and " -1 " together to read as " $\mathrm{L}^{-1}$ " 